(RESEARCH ARTICLE)

\title{
Relative economic value estimates of Guinea fowl (Numida meleagris) production traits.
}

\author{
Sadick Abdul Muumin 1, *, Bonsu Fritz Ramseyer Karikari 1, Abunyuwah Isaac ${ }^{2}$ and Annor Serekye Yaw 1 \\ ${ }^{1}$ Department of Animal Science Education, University of Education, Winneba. P. O. Box 40. Mampong Ashanti. Ghana. \\ 2 Department of Agricultural Economics and Extension Education, University of Education, Winneba. P. O. Box 40. \\ Mampong Ashanti. Ghana.
}

Publication history: Received on 15 July 2020; revised on 22 July 2020; accepted on 25 July 2020

Article DOI: https://doi.org/10.30574/wjarr.2020.7.1.0265

\begin{abstract}
The high demand for the Guinea fowl meat and products due to its delicacy, demands improvement in the bird to meet consumer needs. The inability to develop breeding goals to improve the Guinea fowl in Africa is as a result of limited information on the relative economic values of traits in the bird. This study was carried out to estimate the relative economic values of body weight, survival, reproduction, feed intake, growth rate and docility in the Guinea fowl. Average values from secondary data were used in estimating the relative economic values of the Guinea fowl traits. Data were analysed using computer simulation models in Microsoft Excel spreadsheet of Windows 10. When genetic standard deviation was used in estimating the relative economic values, body weight (55.45\%) and reproduction (25.28\%) emerged as the most important traits that contributed to the profit of the farmer. Similar magnitudes and directions were maintained (52.35\% and $26.07 \%$ ) for body weight and reproduction traits respectively as the most important traits when medication was set to zero. It was concluded from the study that, hen-day egg production and growth rate were the most important traits that contributed highly to profit. It was recommended that growth rate and reproduction traits should be included in the breeding goals of Guinea fowl breeding for improved profitability.
\end{abstract}

Keywords: Guinea Fowl; Breeding Goals; Reproduction Trait; Growth Rate Trait.

\section{Introduction}

Commercial Guinea fowl has gained much popularity in most countries such as United States, France, Belgium and Italy [1]. France is the highest leading producer and consumer of Guinea fowl meat in the whole of Europe with Italy taking the second position [2]. The success of these countries in the production of the Guinea fowl is as a result of careful selection of economically valuable traits that has led to considerable increase in performance of the birds in these countries [3].

The economic value of a trait represents the change in profit of a farmer per unit product output resulting from a unit change in the performance of the trait under consideration while other traits remain constant [4]. In deriving economic value of traits, the future expectations of prices of the traits should be considered [5]. Relative economic value (REV) depicts the economic values of respective traits in relation to other traits. The REV can also be termed as estimated breeding value for profit (Pebv) [6].

The economic values of traits in various livestock species have been estimated. The economic values in the Dutch broiler chicken has been estimated in the Netherlands [4] and for quality chicken in China [7]. The REVs for linear and quadratic bio economic objectives in the commercial broiler has been considered in the United States [8]. However, there is limited literature on the REVs of traits in the Guinea fowl in Ghana. The estimation of the REVs of traits would serve as the basis

\footnotetext{
* Corresponding author: Sadick Abdul Muumin Email: amsadick@yahoo.com
} 
for the development of Guinea fowl breeding goals which aids in the improvement of the bird and increase the financial returns of Guinea fowl breeders and farmers.

The study was conducted to estimate the REVs of body weight, survival, reproduction, feed intake, growth rate and docility traits in the Guinea fowl using genetic standard deviation and find the effect of medication on REVs of traits.

\section{Material and methods}

\subsection{Study location and duration}

The study was conducted at the poultry section of the Department of Animal Science farm of University of Education, Winneba, Mampong campus. Mampong lies in the transitional zone between the Guinea savannah zone of the north and the tropical rain forest of the south of Ghana on latitude 07ㅇ 04' North and longitude 01ㅇ 24 West and an altitude of about $457 \mathrm{~m}$ above sea level. The study lasted for a period of six months.

\subsection{Data collection and Analysis}

Secondary source data regarding production and reproduction potential of Guinea fowl together with husbandry management practices of the Guinea fowl unit of Animal Science farm of University of Education, Winneba, were utilized in order to optimize the computer model. Computer simulation model developed in Microsoft Excel of windows 10 was used to analyze the data.

The derivation of REVs followed the following steps in accordance with [9].

- The specification of the breeding, production, processing and marketing systems.

- Identification of source of income and expenses.

- Identification of biological traits influencing income and expenses.

- Derivation of economic values of each trait.

- Calculation of REV of each trait.

\subsubsection{Specification of the breeding, production, processing and marketing systems}

Computer models were developed for the production cycle of a family of Guinea fowl. The family of Guinea fowl consisted of a cock and five hens. The model was developed in Microsoft Excel spreadsheet of Windows 2010. The model calculations were based on the cycle of production of the breeding hen which was assumed to last for 24 months and the age at first laying was six months. The model output consisted of the profit to be derived from the sale of Guinea keets, smaller eggs, larger eggs, culled cocks and hens.

A model flow diagram was used to describe the production system as shown in Fig.1 and assumptions used in constructing the Guinea fowl model is shown in Table 1.

Table 1 Average values and assumptions used to construct the Guinea fowl model

\begin{tabular}{lll}
\hline Parameter & Acronym & Mean Value \\
Flock structure & & 2 \\
Number of breeding cock keets & ckNum & 8 \\
Number of breeding hen keets & chNum & 68 \\
Pre-brooding survival (\%) & SPreBrood & 92 \\
Post-brooding survival (\%) & SPOBrood & 180 \\
Age at first egg (days) & AFE & 63 \\
Hen day egg production (\%) & HDEP & 210 \\
Duration of egg laying (days) & DurEL & 40 \\
Proportion of small sized eggs (\%) & \%SE & 2 \\
\hline
\end{tabular}




\begin{tabular}{|c|c|c|}
\hline Proportion of medium sized eggs (\%) & $\% \mathrm{ME}$ & 55 \\
\hline Proportion of large sized eggs (\%) & $\% \mathrm{LE}$ & 5 \\
\hline Egg fertility rate $\%$ & EFR & 57 \\
\hline Hatchability \% & Hat & 51 \\
\hline Length of life cycle of hens (days) & hLCyc & 540 \\
\hline \multicolumn{3}{|l|}{ Production variables } \\
\hline Hatch weight of hen keets (g) & hWHat & 24.77 \\
\hline Hatch weight of cock keets (g) & cWHat & 24.35 \\
\hline Weaning weight of hen keets (g) & hWWT & 380.60 \\
\hline Weaning weight of cock keets (g) & cWWT & 411.70 \\
\hline PreBrooding daily gain of keets (g/day) & PreBroodDG & 6.3 \\
\hline Post-brooding Daily gain of birds (g/day) & POBroodDG & 9.6 \\
\hline Breeding weight of cock (g) & cMWT & 1714 \\
\hline Breeding weight of hens (g) & hMWT & 1695 \\
\hline \multicolumn{3}{|l|}{ Management variables } \\
\hline Days from hatch to end of brooding (days) & dHatBrood & 56 \\
\hline Days from Post-brooding to maturity (days) & dPOBroodMat & 112 \\
\hline Days from maturity to end of egg lay (days) & dMatLay & 365 \\
\hline Brooding daily feed intake of birds (g/bird ) & DFIBrood & 14.8 \\
\hline Growers Daily feed intake of (g/bird ) & GrowDFI & 71.3 \\
\hline Breeders Daily feed intake of (g/bird ) & BreedDFI & 96.20 \\
\hline Docility & $\mathrm{D}$ & 3.26 \\
\hline \multicolumn{3}{|l|}{ Cost and Returns Variables } \\
\hline Purchase price of breeding keets (GHC/animal) & bkPP & 6.0 \\
\hline Number of slated floor cages used & NumCag & 1 \\
\hline Cost of one slated floor cage (GHC) & CosCag & 46 \\
\hline Equipment cost per cage (GHC) & EquiCosCag & 17.77 \\
\hline Labour cost/head/day (GHC) & CosLabD & 0.33 \\
\hline Number of labour used & NumLab & 1 \\
\hline Annual cost of stationery (GHC) & CosStat & 2.20 \\
\hline Brooder Feed cost per kg (GHØ) & BroodFC & 2.39 \\
\hline Grower Feed cost per kg (GHC) & GOFC & 1.96 \\
\hline Layer Feed cost per kg (GHC) & LayFC & 1.79 \\
\hline Brooding Feed cost per bird (GHC) & BroodFCb & 1.98 \\
\hline Growing Feed cost per bird (GHC) & $\mathrm{GOFCb}$ & 15.65 \\
\hline Laying Feed cost per bird (GHC) & LayFCb & 36.16 \\
\hline Quantity of water used per day (Litres) & WatQuant & 1 \\
\hline Cost of water (GHC/lit) & CosWater & 0.003 \\
\hline Incubator charge per egg set (GHC) & IncuChar & 0.4 \\
\hline
\end{tabular}




\begin{tabular}{|c|c|c|}
\hline Selling price per unit smaller eggs & PriceSE & 0.5 \\
\hline Selling price per unit larger egg & PricelE & 0.6 \\
\hline $\begin{array}{l}\text { Brooding cost of veterinary service charges and } \\
\text { drugs/bird (GHC) }\end{array}$ & BroodVetChar & 0.70 \\
\hline $\begin{array}{l}\text { Growing cost of veterinary service charges and } \\
\text { drugs/bird (GHC) }\end{array}$ & GrowVetChar & 2.28 \\
\hline $\begin{array}{l}\text { Laying cost of veterinary service charges and } \\
\text { drugs/bird (GHC) }\end{array}$ & LayVetChar & 4.23 \\
\hline Selling price/ keet (GHC) & kSelPrice & 7.00 \\
\hline Selling price/g cock (GHC) & cgmPrice & 0.02 \\
\hline Selling price/g hen $(\mathrm{GH} \not)$ & hgmPrice & 0.02 \\
\hline Discount rate (\%) & $d$ & 16 \\
\hline
\end{tabular}

Feed was assumed to be provided in the form of formulated diet. The assumption was that; a labourer would spend 1 hour per day on the birds. Treatment of sick birds against diseases was assumed be carried out by a veterinary officer. Marketing of eggs, keets and culled bird were assumed to be sold at the farm gate at the end of the production cycle.

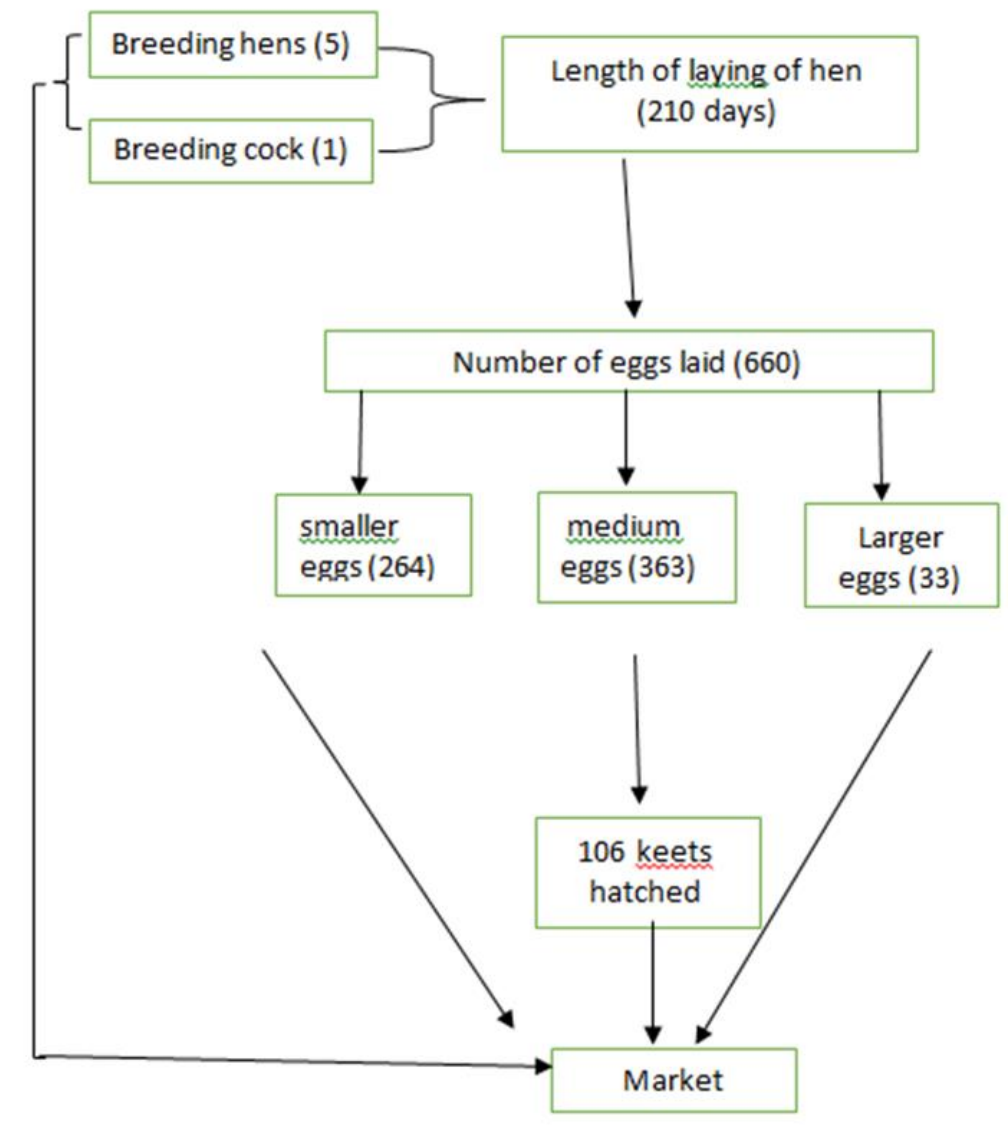

Figure 1 Model flow diagram from five breeding hens and a breeding cock derived from average values of traits.

\subsubsection{Identification of source of income and expenses}

Profit was defined as the difference between income (I) and cost (C). Income was derived from the sale of culled cocks and hens, small and larger eggs and day-old keets.

The profit derived from the birds was expressed as a function of the traits and animals as; 
$P=\sum_{i=1}^{m} \sum_{j=1}^{n}(V i j-C i j)-K$

Where $\mathrm{P}$ is the profit

$\mathrm{Vij}=$ revenue from the ith trait of the jth class of animal

Cij= Cost from the ith trait of the jth class of animal

$\mathrm{K}=$ fixed cost

$\mathrm{i}=1,2,3$

$j=1,2,3 \ldots \ldots \ldots$

For each class of product, income was calculated as the sum of the product of the number of birds or eggs sold and the price per individual. Variable expenses were derived from cost of feed, water, veterinary service charges, incubator charges and drugs and fixed costs included cost of breeding stock, slated floor cages, labour, equipment, and stationery.

\subsubsection{Determination of biological traits influencing income and expenses}

The life cycle model was used to estimate economic values of the following traits;

- Survival (SPreBrood, SPOBrood)

- Reproduction (AFE, HDEP, EFR, Hat)

- Body weight (cWHat, hWHat, cWWT, hWWT, cMWT, hMWT)

- Growth rate (PreBroodDG, POBroodDG)

- Feed intake (PreBroodDFI, GrowDFI, BreedDFI)

- Behavioural (Docility)

Table 2 Biological traits influencing income and expenses.

\begin{tabular}{lll}
\hline Product or activity & Class of bird & Traits in breeding objective \\
\hline Income & Cock Keets & cWHat SPreBrood, KBroodDG, \\
& Hen Keets & hWHat SPreBrood, KBroodDG, \\
& Grower Cock & cWWT, SPOBrood, POBroodDG, \\
& Grower Hen & hWWT, SPOBrood, POBroodDG \\
& Breeder Cock & cMWT \\
Breeder Hen & hMWT, EFR, Hat, HDEP \\
Expenses & Cock keets & SPreBrood, cWHat, cMWT, DFIPreBrood, GrowDFI \\
Feed intake & Hen keets & SPreBrood, hWHat, cWWT, hMWT, DFIPreBrood, \\
& Grower cock & GrowDFI, cMWT \\
& Grower hens & hMWT, GrowDFI, \\
Breeder cock & BreedDFI \\
Breeder hen & BreedDFI \\
Cock keets & SPreBrood \\
Veterinary charges and & SPreBrood \\
drugs & Hen keets & SPOBrood \\
& Grower cock & SPOBrood \\
Grower hen & cMWT, EFR, Hat, HDEP \\
& Breeder cock & hMWT, EFR, Hat, HDEP \\
& Breeder hen &
\end{tabular}




\subsubsection{Calculation of economic value of each trait}

A partial budgeting approach was used in estimating the economic values of the traits in accordance with [9]. The economic value of each trait was determined as the marginal profit per bird per year per unit improvement in the genetic merit of each trait while other traits remained constant [4]. The economic values of the traits were calculated by the difference between the profit per year per bird and the profit per year per bird when a trait was improved by $1 \%$ whiles other traits were held constant [9].

Docility scores from [10] were used in estimating the economic values for docility. Prices were regressed on the docility scores to estimate the economic value of docility.

\subsubsection{Calculation of REVs of each trait}

The REV's of each trait was calculated by multiplying the discounted economic value by the genetic standard deviation and dividing each individual value by the absolute values of all traits in the breeding objective and expressing it as a percentage [9].

$R E V=\left[\frac{e v_{i} \times O_{g}^{\prime}}{\sum\left(e v_{i} \times O_{g}^{\prime}\right)}\right] \times 100$

Where evi = economic value of the ith trait and O'g =genetic standard deviation.

A sensitivity analysis was carried out on REVs to find out the effect of the removal of medication cost from the model. This was done because Guinea fowl is a hardy bird and resistant to most poultry diseases $[11,12,13,14]$.

\section{Results}

\subsection{Estimation of REVs using Genetic standard deviation (Ơg)}

The combined body weight traits had the highest REV (55.45\%), followed by reproduction traits (25.28\%), feed intake (13.07\%), survival (5.34\%) and growth rate (0.82\%) (Fig. 4.1). Behavioural trait was the least important with REV of $0.11 \%$. This implies that, on the average body weight had the highest REV and hence contributed highly to profit gained as compared to other traits. This was followed by reproduction, feed intake, survival, growth rate in that order with docility making the least contribution to profit as shown in figure 2.

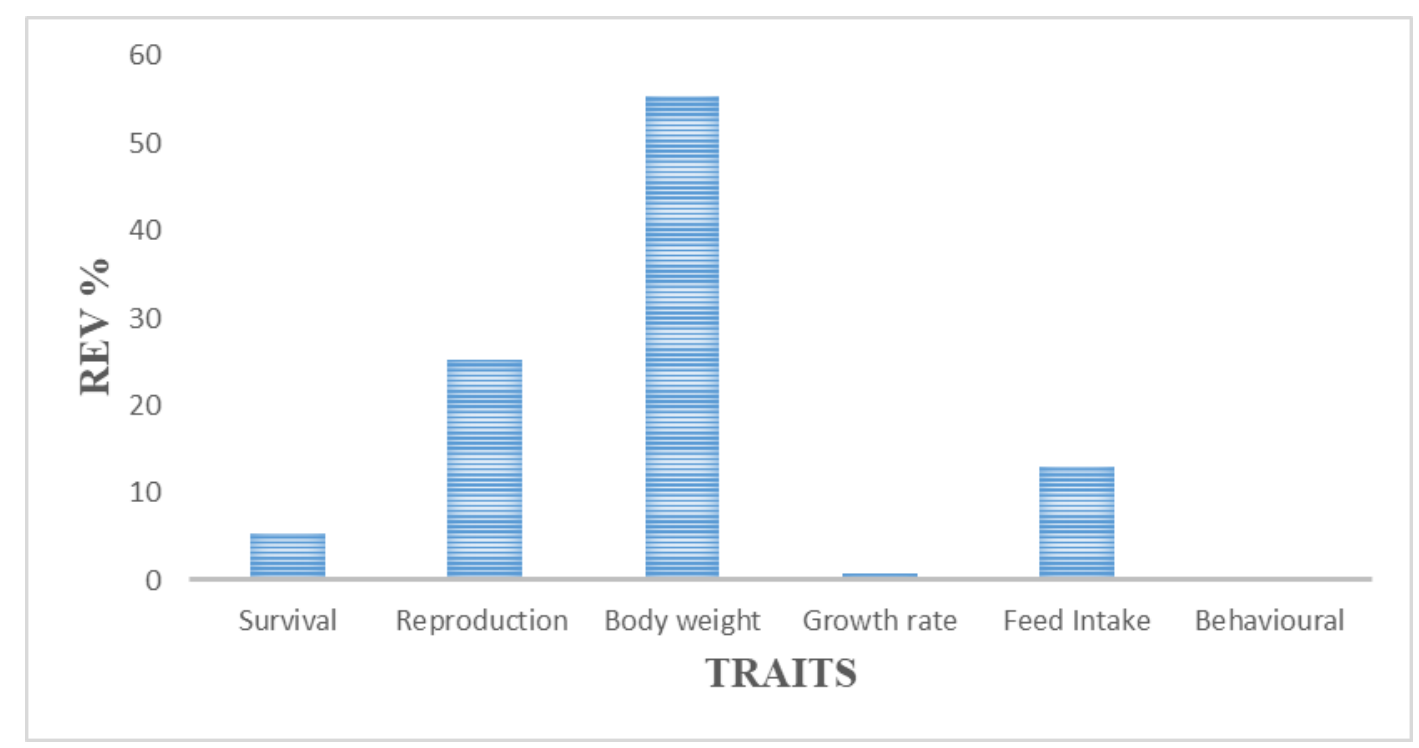

Figure 2 Combined REVs when Ơg was used 


\subsection{Estimation of REV when Medication was set to zero and Ơg was used.}

When medication was set to zero and genetic standard deviation was used in estimating the REVs, body weight traits emerged as the most important trait (52.35\%) followed by reproduction (26.07\%), feed intake (13.79\%), survival $(4.83 \%)$ and growth rate traits (2.92\%). Behavioural trait was the least important (0.11\%) (Fig. 3).

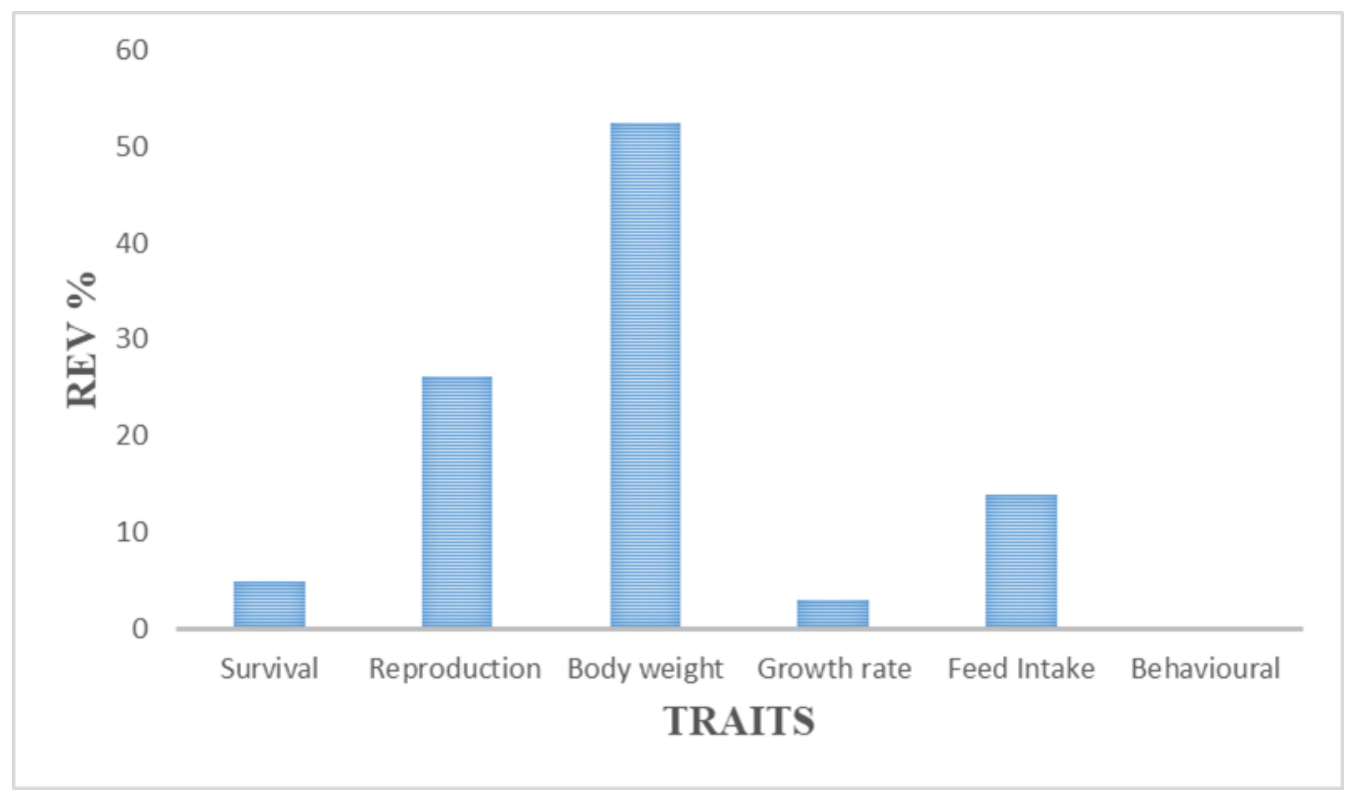

Figure 3 Combined REV of traits when medication was set to zero and $O_{\mathrm{g}}^{\prime}$ was used

\section{Discussions}

\subsection{Estimation of REVs when medication was included in the Model.}

This study confirms earlier reports from the study of [9] in grasscutter in Ghana and cattle in Slovakia [15] respectively that, body weight and reproduction traits contributed highly to profit. The current study is also in agreement with studies conducted on Rayeni cashmere goats which stated production traits and reproduction traits as the most important traits [16]. In China, [7] reported body weight traits and reproduction traits as the most important traits in chicken breeding. Also, production and reproduction traits in pigs breeding have been reported to be traits with higher economic values [17]. However, this study contradicts the previous reports by [18] who reported survival to be the highly contributing trait to profit with growth traits contributing the least to profit. The contradiction is resulting from the use of REV in ranking in the present studies whiles in the previous study ranking of the traits were based on absolute economic values.

Behavioural trait contributed the least to profit. This observation is consistent with the report by [9] in grasscutter. The explanation is that most farmers and consumers pay little attention to the behaviour of farm animals during purchase [9].

\subsection{Effect of Medication on REV of Traits}

When medication was set to zero, there was a slight change in magnitude in the REVs. There was an increase in magnitude in the REVs of all reproduction traits. Similar observation was made by both [18] and [9] who reported increased reproductive traits when feed cost was set to zero in cattle and grasscutter respectively in Ghana. Body weight emerged as the most important trait followed by reproduction, reproduction, survival and growth rate. Behavioural trait was the least important trait. Variations introduced when medication was set to zero were less and did not affect the ranking order of the combined REVs of traits. Similar reports have been made in grasscutter [9] with body weight still being the most important trait after setting feed cost to zero.

In all, growth rate and reproduction (hen-day egg production) were the suggested traits to be included in the Guinea fowl breeding goals. Reproductive trait (Hen-day egg production) is an important trait because it is highly positively correlation with survival [19]. Again growth rate has high positive correlation with feed intake and body weight. This is 
because higher body weight depends on the growth rate, which in turn depends on the feed intake. Again survival is dependent on growth rate [9].

\section{Conclusion}

Body weight and reproduction traits were the most important traits that contributed to profit when genetic standard deviation was used in estimating the REVs of traits. Setting medication costs to zero had no significant influence on the REVs of traits. Reproduction (Hen-day egg production) and growth rate are traits recommended to breeders to be inculcated into the Guinea fowl breeding objectives. Further research should be conducted using regression analysis in estimating the economic values of traits in the Guinea fowl instead of the partial budgeting approach.

\section{Compliance with ethical standards}

\section{Acknowledgments}

The authors are grateful to the Department of Animal Science Education, Faculty of Agriculture Education, University of Education, Winneba for providing facilities for this study.

\section{Disclosure of conflict of interest}

Authors have declared that, no conflict of interests exist.

\section{References}

[1] Nahashon SN, Aggrey SE, Adefope NA, Amenyenu A and Wright D. (2006). Modeling growth characteristics of meat-type Guinea fowl. Journal of Poultry Science, 85(5), 943.

[2] Kokoszyński D, Bernacki Z, Korytkowska H and Piotrowska K. (2011). Effect of age and sex on slaughter value of guinea fowl (Numida meleagris). Journal of Central European Agriculture, 12(2), 255-266.

[3] Zagbede AG, Annor SY, Duodu A and Arhin E. (2019). Effects of Strain and Non-genetic Factors on the Egg Qualities and Carcass Characteristics of Indigenous Guinea Fowl (Numida meleagris). International Journal of Applied Agricultural Sciences, 5(2), 39-44.

[4] Jiang X, Groen AF and Brascamp EW. (1970). Economic Values in Broiler Breeding. Poultry Science, 77, $934-943$.

[5] Kulak K, Wilton J, Fox G and Dekkers J. (2003). Comparisons of economic values with and without risk for livestock trait improvement. Livestock Production Science, 79, 183-191.

[6] Van Graan A, Olivvier W and Herselman M. (2018). Relative Econpmic Value for Merino Sheep in South Africa. Conference Paper, (September), 1-4.

[7] Chaowu Y, Xiaosong J, Hurui D, Qingyun L, Zengrong Z and Mohan Q. (2019). Economic Values for Production Systems of Quality Chicken in China. Modern Agricultural Science and Technology, 2(2), 36-45.

[8] Akbar MK, Harris DL and Arboleda R. (1986). Development of the Relative Economic Weights for Linear and Quadratic Bioeconomic Objectives in Commercial Broilers. Roman L. Hruska U.S. Meat Animal Research Center, 444.

[9] Annor S, Ben A and Cassady J. (2014). REV estimates of grasscutter production traits. Int. J. Livest. Prod. Household, 138-140.

[10] Duodu A, Annor SY, Kagya-Agyemang JK and Kyere CG. (2018). Influence of Strain on Production and Some Other Traits of Indigenous Guinea Fowls (Numida meleagris) in Ghana. Current Journal of Applied Science and Technology, 30(2), 1-7.

[11] Agbolosu AA, Teye G and Naandam J. (2011). Comparative study of growth and laying performance of indigenous layer guinea fowls (Numida meleagris) from upper east, upper west and northern regions of Comparative study of growth and laying performance of indigenous layer guinea fowls (Numida meleagris). Agric. Biol. J. N. Am, 3(9), 354-359 1.

[12] Ebegbulem VN. (2018). Prospects and challenges to guinea fowl (Numida meleagris) production in Nigeria. Int J Avian \& Wildlife Biol, 3(3), 182-184. 
[13] Moreki JC. (2009). Guinea Fowl Production. Reach Publishers, Wandsbeck, South Africa, 3631, 7-31.

[14] Moreki JC and Radikara MV. (2013). Challenges to Commercialization of Guinea Fowl in Africa. Int. J. Sci. Res, 2(11), 436-440.

[15] Krupa E, Wolfová M, Peškovičová D, Huba J and Krupová Z. (2005). Economic values of traits for Slovakian Pied cattle under different marketing strategies. Czech J. Anim. Sci, 483-492.

[16] Borzi NK, Mehrgardi AA, Vatankhah M and Fozi MA. (2017). Determination of economic values for some important traits of Rayeni cashmere goats reared under pasture system. Journal of Livestock Science and Technologies, 5(1), 51-58.

[17] De Vries AG. (1989). Selection for production and reproduction traits in pigs. Livestock Production Science, 21, 49-66, 164.

[18] Annor SY. (2000). Development of a breeding objective for beef cattle in Ghana. Master's Thesis. Massey University, 150-261.

[19] Saina H. (2005). Guinea fowl (Numidia meleagris) production under smallholder farmer management in Guruve District, Zimbabwe. Master's Thesis, University of Zimbabwe, 7-8.

\section{How to cite this article}

Sadick AM, Bonsu FRK, Abunyuwah I and Annor SY. (2020). Relative economic value estimates of Guinea fowl (Numida meleagris) production traits. World Journal of Advanced Research and Reviews, 7(1), 273-281. 